\title{
Tissue kallikrein-modified human endothelial progenitor cell implantation improves cardiac function via enhanced activation of akt and increased angiogenesis
}

\author{
Yuyu Yao ${ }^{1}$, Zulong Sheng ${ }^{1}$, Yefei Li ${ }^{1}$, Cong Fu ${ }^{1}$, Genshan Ma', Naifeng Liu', Julie Chao ${ }^{2}$ and Lee Chao ${ }^{2}$
}

Endothelial progenitor cells (EPCs) have been shown to enhance angiogenesis not only by incorporating into the vasculature but also by secreting cytokines, thereby serving as an ideal vehicle for gene transfer. As tissue kallikrein (TK) has pleiotropic effects in inhibiting apoptosis and oxidative stress, and promoting angiogenesis, we evaluated the salutary potential of kallikrein-modified human EPCs (hEPCs; Ad.hTK-hEPCs) after acute myocardial infarction (MI).

We genetically modified hEPCs with a TK gene and evaluated cell survival, engraftment, revascularization, and functional improvement in a nude mouse left anterior descending ligation model. hEPCs were manipulated to overexpress the TK gene. In vitro, the antiapoptotic and paracrine effects were assessed under oxidative stress. TK protects hEPCs from oxidative stress-induced apoptosis via inhibition of activation of caspase-3 and -9, induction of Akt phosphorylation, and secretion of vascular endothelial growth factor. In vivo, the Ad.hTK-hEPCs were transplanted after MI via intracardiac injection. The surviving cells were tracked after transplantation using near-infrared optical imaging. Left ventricular (LV) function was evaluated by transthoracic echocardiography. Capillary density was quantified using immunohistochemical staining. Engrafted Ad.hTK-hEPCs exhibited advanced protection against ischemia by increasing LV ejection fraction. Compared with Ad.Null-hEPCs, transplantation with Ad.hTK-hEPCs significantly decreased cardiomyocyte apoptosis in association with increased retention of transplanted EPCs in the myocardium. Capillary density and arteriolar density in the infarct border zone was significantly higher in Ad.hTK-hEPC-transplanted mice than in Ad.Null-hEPC-treated mice. Transplanted hEPCs were clearly incorporated into $\mathrm{CD} 31^{+}$capillaries. These results indicate that implantation of kallikrein-modified EPCs in the heart provides advanced benefits in protection against ischemia-induced MI by enhanced angiogenesis and reducing apoptosis.

Laboratory Investigation (2013) 93, 577-591; doi:10.1038/labinvest.2013.48; published online 18 March 2013

KEYWORDS: endothelial progenitor cells; gene therapy; myocardial infarction; tissue kallikrein

Stem cell transplantation shows great promise as a tool in regenerative medicine as it offers a promising means of improving cardiac function after myocardial infarction (MI). Accumulating evidence suggests that bone marrow (BM)-derived endothelial progenitor cells (EPCs) participate in the process of postnatal neovascularization and tissue repair. ${ }^{1,2}$ After ischemia, implantation of EPCs into ischemic myocardium can home to sites of damaged myocardium and enhance recovery. ${ }^{3}$ However, most implanted cells are lost to ischemia, apoptosis, and inflammatory alterations. In addition, it was reported that there was significant loss of grafted cells within $24 \mathrm{~h}$ after transplantation. ${ }^{4}$ Thus, the survival of transplanted stem cells within the ischemic myocardium remains as a major limitation.

Genetic modification of stem cells may represent an important strategic advancement in regenerative medicine. Dzau's group was the first to show that mesenchymal stem cells (MSCs) overexpressing the antiapoptotic gene Akt1 became more resistant to apoptosis in vitro and in vivo. ${ }^{5}$ Strategies to lessen apoptosis are important for stem cell survival, leading to cardiac regeneration in the infarcted myocardium.

The tissue kallikrein (TK)-kinin system components have been identified in the cardiovascular system. ${ }^{6}$ The serine

\footnotetext{
${ }^{1}$ Department of Cardiology, Zhongda Hospital, Medical School of Southeast University, Nanjing, China and ${ }^{2}$ Department of Biochemistry and Molecular Biology, Medical University of South Carolina, Charleston, SC, USA

Correspondence: Dr Y Yao, PhD, Department of Cardiology, Zhongda Hospital, Medical School of Southeast University, 87 Dingjiaqiao, Jiangsu, Nanjing 210009 , USA. E-mail: yaoyuyunj@hotmail.com

Received 15 June 2012; revised 11 February 2013; accepted 12 February 2013
} 
protease TK specifically processes low-molecular-weight kininogen to produce the potent vasoactive peptides bradykinin (BK) and Lys-BK (kallidin). ${ }^{7}$ Previous studies showed that TK stimulates the activation of Akt in vitro and in vivo. ${ }^{8,9}$ Local delivery of adenovirus carrying the human TK gene (Ad.hTK) inhibits GSK-3 $\beta$ and caspase- 3 activity by Akt activation in the ischemic myocardium. ${ }^{10}$ Moreover, results from different research groups consistently showed that TK promotes vessel growth after MI by increasing the number of circulating EPCs and enhancing EPCs differentiation, migration, tube formation capacity, and survival through a kinin-B2 receptor-dependent Akt signaling pathway. ${ }^{8,11}$ These data highly suggest that the KKS has multifactorial roles in tissue repair after MI.

The current study was designed to test the hypothesis that EPCs genetically modified with the kallikrein gene exert advanced beneficial effects in protection against acute ischemic injury by suppression of apoptosis and inducing angiogenesis using a mouse MI model.

\section{Materials and methods \\ Cell Culture and Characterization of Human EPCs}

Human umbilical cord blood was obtained from Zhongda Hospital. All selected pregnant women were healthy and signed an informed consent form. The obtained human umbilical cord blood was diluted to a 1:1 ratio in phosphatebuffered saline, and the mononuclear cell (MNC) fraction was obtained from a Lymphoprep density gradient (Sigma, St Louis, MO, USA) after centrifugation. The cell pellet was suspended in growth factor-supplemented EGM-2 medium (Lonza, Walkersville, MD, USA). After removing unbound cells at $96 \mathrm{~h}$, the bound cell fraction was then maintained in culture using EGM-2. Spindle-shaped cells were observed after 7 days. Colonies of endothelial-like cells grew until confluent. Cells were passaged at a split ratio of 1:2. Passages 3-4 human EPCs (hEPCs) were used in the study.

Cells were incubated with acLDL-Dil for $4 \mathrm{~h}$ at $37^{\circ} \mathrm{C}$. Lectin binding was analyzed using FITC-conjugated UEA-1lectin (Sigma) after 7 days in culture. Flow cytometry was utilized for analyzing the expression of endothelial lineage marker VEGFR2 (KDR; BD Biosciences, Massachusetts, USA), progenitor cell marker CD34, and BK B2 receptor (BD Biosciences).

\section{Preparation of Replication-deficient Adenoviral Vectors and Generation of TK- Modified hEPCs}

Adenovirus harboring the human TK cDNA (Ad.hTK) under the control of the cytomegalovirus enhancer/promoter or adenoviral vector alone (Ad.Null) were constructed and prepared as described previously. ${ }^{12}$ hEPCs were seeded into six-well plates. After $48 \mathrm{~h}$, cells were transduced with Ad.hTK or Ad.Null at multiplicity of infection of 50 for $12 \mathrm{~h}$. Expression and localization of human TK in EPCs after Ad.hTK transduction were identified immunocytochemically using a rabbit anti-kallikrein antibody.

\section{Detection of Apoptosis Caused by $\mathrm{H}_{2} \mathrm{O}_{2}$}

In this study, we incubated cultured hEPCs with $\mathrm{H}_{2} \mathrm{O}_{2}$, a relatively stable reactive oxygen compound used to mimic oxidative stress in vitro. Ad.Null-hEPCs, Ad.hTK-hEPCs and non-Adv-hEPCs were seeded in six-well plates cultured with endothelial cell basal medium-2 (EBM2, 1\% serum, growth-factor-free). Cells were treated with $\mathrm{H}_{2} \mathrm{O}_{2}(0.5 \mathrm{mM})$ for $12 \mathrm{~h}$. hEPCs apoptosis was evaluated with annexin V-FITC/propidium iodide (PI) Apoptosis Detection Kit (Biouniquer Technology CO, Hangzhou, China). After treatment, the adherent and non-adherent cells were collected. The cells were then stained with annexin-V-FITC and PI in binding buffer for $15 \mathrm{~min}$. Flow-cytometric analyses were performed on a FACS flow cytometer (Becton Dickinson, Germany), and the data were analyzed by the Cell Quest analysis program.

hEPCs cultured on a cover glass were fixed with $4 \%$ paraformaldehyde solution. Terminal deoxynucleotidyl transferase-mediated dUTP nick end labeling (TUNEL, In Situ Cell Death Detection Kit; Roche, Mannheim, Germany) reaction mixture was added to each sample for $1 \mathrm{~h}$ at $37^{\circ} \mathrm{C}$. These samples were analyzed by fluorescence microscopy as described previously. ${ }^{13}$

The activities of caspase- 3 and caspase- 9 were measured using colorimetric assay kits (KeyGen Biotech Co, Ltd, Nanjing, China) according to the manufacturer's instructions. The optical density of each well was measured at $405 \mathrm{~nm}$ using a microplate reader. Activity of caspse-3/9 was expressed in arbitrary absorbance units (absorbance at a wavelength of $405 \mathrm{~nm}$ ).

Ad.Null-hEPCs, Ad.hTK-hEPCs and non-Adv-hEPCs with $\mathrm{H}_{2} \mathrm{O}_{2}$ were cultured with EBM2 media containing 1\% FBS for 1 day. Then media was collected, and protein levels of VEGF were determined by Human VEGF QuantiGlo ELISA Kit (R\&D Systems, Minneapolis, MN, USA) according to the manufacturer's instructions.

\section{Western Blot Analysis}

After treatment, the cells were lysed with cell lysis buffer and centrifuged at $12000 \mathrm{~g}$ for $30 \mathrm{~min}$ at $4{ }^{\circ} \mathrm{C}$. The supernatant was removed, and protein concentrations were measured by BCA assay (Pierce, Rockford, IL, USA). Western blot analysis was performed using cytosolic fractions to detect cleaved caspase- 3 and the total and phosphorylated forms of Akt (Cell Signaling; Danvers, MA). GAPDH was used as a loading control in western blot analysis (Advanced Immunochemical, Long Beach, CA, USA). After primary antibody incubation, the blot was incubated with appropriate secondary anti-IgG horseradish peroxidase conjugate. The membrane was washed and developed with SuperSignal chemiluminescent substrate (Pierce).

\section{In Vitro Experiments with the Conditioned Medium}

To mimic ischemic injury in vitro, hypoxia was achieved by placing the cells in a Modular Incubator Chamber 
(Billups-Rothenberg; Del Mar, CA, USA) according to the manufacturer's instructions. Conditioned medium was generated as follows: cell cultures containing isolated (90\%) Ad.TK-hEPCs and Ad.Null-hEPCs were fed with EBM2 and incubated for $12 \mathrm{~h}$ under hypoxia. The medium was then collected and used for in vitro experiments.

Cardiomyocytes were isolated from 1-2-day old neonatal Sprague-Dawley rats (Animal Center, Yangzhou University, China) as previously described. ${ }^{9}$ We used a 60 -min preplating procedure to reduce the number of non-myocytes in the culture. Then, after 3 days in culture, the isolated rat neonatal cardiomyocytes were cultured in supernatant collected from either Ad.TK-hEPCs or Ad.Null-hEPCs cultures treated as stated above, after which $\mathrm{H}_{2} \mathrm{O}_{2}$ was directly added to the culture medium to form a final concentration of $200 \mu \mathrm{M}$. The cardiomyocytes were then incubated for $12 \mathrm{~h}$. Following incubation, the cells were collected and the number of apoptotic cells was determined using Hoechst 33324 staining.

\section{hEPC Labeling}

To evaluate the incorporation of hEPCs into ischemic myocardium, Ad.Null-hEPCs and Ad.hTK-hEPCs were trypsinized and then resuspended at a concentration of $1 \times 10^{6} / \mathrm{ml}$ in EBM2 (serum-free, growth-factor-free). Sterile 1, $1^{\prime}$-dioctadecyl-3,3,3',3'-tetramethylindodicarbocyanine, 4 chlorobenzenesulfonate salt (DiD; Invitrogen, CA, USA) celllabeling stock solution was added to cell suspension $(5 \mu \mathrm{l} / \mathrm{ml})$ and then cells were incubated for $20 \mathrm{~min}$ at $37^{\circ} \mathrm{C}$. After labeling, cells were washed three times to remove unbound DiD. Subsequently, the labeled cells were assessed by cell proliferation assay and labeling efficiency measurement or prepared for the subsequent studies.

\section{Nude Mouse Model of Acute MI and Transplantation of Labeled hEPCs}

All animal studies were approved by the Institutional Animal Care and Use Committee of Southeast University. The recipient male nude mice (weight 20-22 g) were anesthetized with intraperitoneal pentobarbital $(45 \mathrm{mg} / \mathrm{kg})$ and intubated. The left anterior descending (LAD) coronary artery was ligated proximally with $8-0$ silk suture via a left thoracotomy incision. Ten minutes later, animals were randomly divided into three groups ( $n=12$ in each group). In two control groups, mice were subjected to either sham surgery or LAD coronary ligation followed by medium injection (mediumtreated group). The third group received $30 \mu \mathrm{l}$ of intramyocardial injections of $5 \times 10^{5}$ Ad.Null-modified hEPCs (Ad.Null-hEPCs-treated group), and the fourth group received the same dose of Ad.hTK-modified hEPCs (Ad.hTKhEPC-treated group). The injections were performed at multiple sites in the border zone of the infarcted heart. After the chests of the animals were sutured, the animals were allowed to recover. Expression and localization of TK in mice ventricles after stem cell delivery were identified immunohistochemically by antibody to TK.

\section{Measurement of Blood Pressure and Cardiac Function}

Blood pressure of conscious mice was measured by a noninvasive computerized tail-cuff method (model BP-2000; Visitech Systems), as described previously. ${ }^{14}$ To ensure that the mice had adapted to the method, the blood pressure was taken 20 times consecutively, and the average measurement was used. All animals at day 7 received a cardiac function evaluation using transthoracic echocardiography before they were killed (Vevo 770TM; VisualSonics, Toronto, Canada). Left parasternal two-dimensional M-mode images under short axis at the level of papillary muscles were recorded by using a $30-\mathrm{MHz}$ linear transducer. LV end-diastolic volume (LVEDV), LV end-systolic volume (LVESV), LV internal diameter at end-diastole (LVIDd), and LV internal diameter at end-systole (LVIDs) were measured at the anterior wall, from the short-axis view, just below the level of the papillary muscle. The LV ejection fraction (LVEF) and LV fractional shortening (LVFS) were calculated with standard M-mode echocardiographic equations $(\mathrm{EF}=(\mathrm{LVEDV}-\mathrm{LVESV})$ 100\%/LVEDV; FS $=($ LVIDd - LVIDs $) \quad 100 \% / L V I D d) . \quad$ All measurements were averaged for five consecutive cardiac cycles and performed by an experienced examiner in a blinded fashion.

\section{Measurement of Malondialdehyde Levels}

Oxidative stress was assessed by measuring the levels of malondialdehyde (MDA). Heart samples were ground into a powder in liquid nitrogen, and cytosolic proteins $(500 \mu \mathrm{g})$ were mixed with $2 \%$ butylated hydroxytoluene and quintanilla reagent and boiled for $15 \mathrm{~min}$. The reaction mixture was centrifuged at $3000 \mathrm{~g}$ for $10 \mathrm{~min}$. The soluble phase was measured with a spectrophotometer at $535 \mathrm{~nm}$ using MDA standards $(0-30 \mu \mathrm{mol} / \mathrm{ml})$ (Sigma).

\section{Histological Analyses}

Cardiac tissues were frozen in optimum cutting temperature compound (Miles Laboratories, Naperville, IL, USA) and sectioned at $5 \mu \mathrm{m}$ on a cryostat (Leica CM1950, Nussloch, Germany). Sections were subjected to Masson's trichrome staining or immunohistochemical analysis using a staining kit (Universal Elite ABC; Vector) according to the manufacturer's instructions. We utilized primary antibodies against CD-31 (Santa Cruz Biotechnology, Santa Cruz, CA, USA) and $\alpha$-smooth muscle actin (Santa Cruz Biotechnology). Capillaries were identified as having a diameter $<20 \mu \mathrm{m}$ and a layer of endothelial cells without smooth muscle cells. Arterioles were identified as having a diameter in the range of $20-100 \mu \mathrm{m}$ with a layer of smooth muscle cells. To determine capillary density and arteriolar density, the number of positive staining was counted in a blind fashion in five fields per section of the peri-infarct zone. A total of five sections per heart were analyzed ( $n=6$ for each group). 
In addition, DNA fragmentation was determined using a TUNEL assay in cryostat sections. The procedure was performed using an in situ cell death detection kit (Roche) according to the manufacturer's instructions. TUNELpositive cardiomyocytes in the ischemic myocardium were carefully distinguished from TUNEL-positive noncardiomyocytes. The ratio of TUNEL-positive cardiomyocytes to the total number of cardiomyocytes was calculated. All sections were counterstained with 4, 6-diamidino2-phenylindole (Sigma), and multiple immunofluorescenceconjugated specimens were evaluated by a confocal microscope (FV-1000; Olympus, Tokyo, Japan).

\section{Optical Imaging Studies}

The optical imaging experiments were performed using a CRi Maestro in-vivo molecular imaging system (CRi, Woburn, MA, USA), which permits coverage in the red, far-red, and NIR spectral regions. Light and cube images were acquired for in-vivo and ex-vivo study, respectively. Recipient nude mice were anesthetized with isoflurane inhalation (1.5\%) and placed in the imaging chamber. In-vivo imaging was performed for each animal either 2 or 7 days post injection, followed immediately by ex-vivo imaging of explanted organs (heart, lung, liver, spleen, and kidney). NIR fluorescent signal average intensities from explanted organs were quantified as counts per second per pixels by software from CRi Maestro. Parameters were set as follows: excitation wavelength coverage $595-800 \mathrm{~nm}$ and emission coverage $660-680 \mathrm{~nm}$.

\section{Statistical Analysis}

For all experiments, data were assessed by using either a Student's $t$-test or Bonferroni's test. Values were expressed as mean \pm s.e.m. All statistical analysis was performed using Statview software (SAS Institute Inc, Cary, NC, USA), with a $P$-value $\leq 0.05$ considered to be statistically significant.

\section{RESULTS}

\section{Characterization of hEPCs}

Human umbilical cord blood-derived MNCs were separated by density-gradient centrifugation. Double staining of FITClectin and acLDL-Dil showed that $>95 \%$ of these hEPCs were able to uptake acLDL-Dil and bind EC-specific lectin (Figure 1a). Using flow cytometry to determine the phenotype of hEPCs, we found that $54.6 \pm 3.7 \%$ were positive for CD34, $50.6 \pm 2.1 \%$ for $\mathrm{KDR}$, and $46.1 \pm 3.7 \%$ for $\mathrm{B} 2$ receptor (Figure 1b). In summary, our data indicate that adherent a

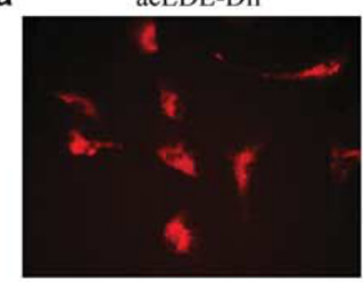

FITC-lectin

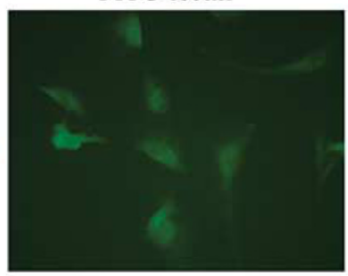

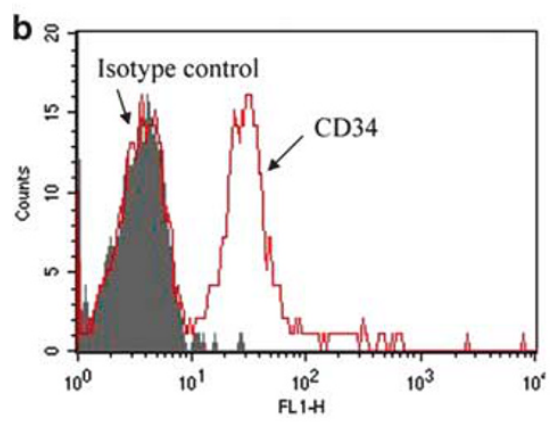
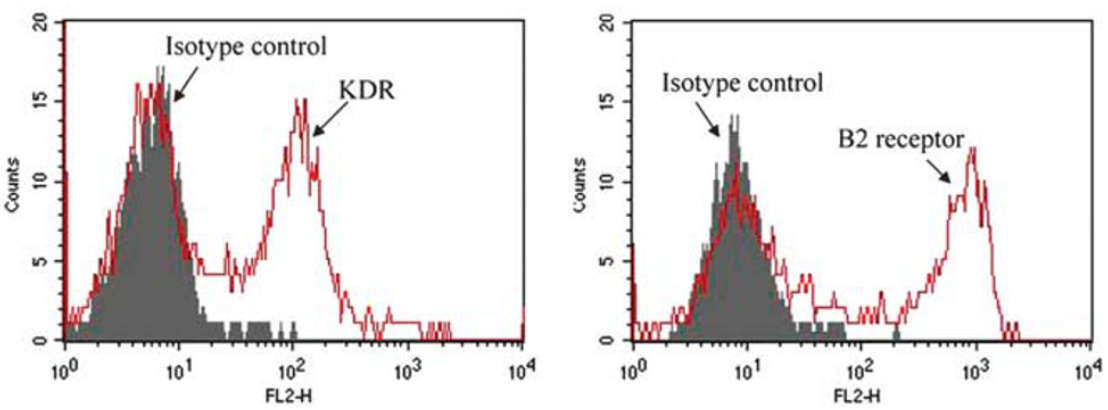

c

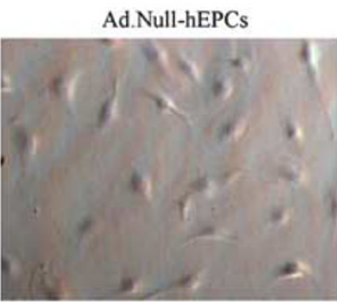

Ad.TK-hEPCs (50MOI)

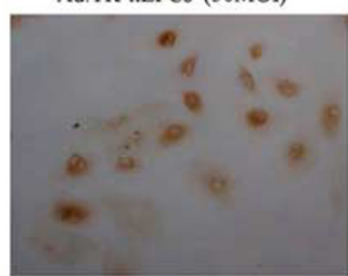

Figure 1 Characterization of cultured hEPCs. (a) At day 7 after hEPC isolation, adherent cells intensively took up acLDL-Dil and bound endothelialspecific lectin-FITC as revealed by fluorescence microscopy. (b) Characterization of hEPCs evaluated by flow cytometry. EPCs were positive for CD34 (55.4\%), CDKDR (49.3\%), and kinin B2 receptor (43.2\%). (c) Expression of human TK in hEPCs after Ad.hTK transduction was confirmed by immunocytochemistry. Original magnification is $\times 200$. 
human umbilical cord blood-derived MNCs at 7 days under these culture conditions contained spindle-shaped cells with characteristics of EPCs.

Increased TK Expression in hEPCs Upon Oxidative Stress After hEPCs were transduced with adenovirus, the expression of human TK was identified by immunocytochemistry (Figure 1c).

To further evaluate the expression of TK in hEPCs in response to oxidative stress, we treated genetically modified hEPCs with $0.5 \mathrm{mM} \mathrm{H}_{2} \mathrm{O}_{2}$ for $12 \mathrm{~h}$. The cell apoptosis rates were detected by flow cytometry (Figure 2a). The apoptosis rate of hEPCs significantly increased after $12 \mathrm{~h} \mathrm{H}_{2} \mathrm{O}_{2}$ treatment $(27.4 \pm 5.5 \%, n=4)$, whereas TK expression protected EPCs from $\mathrm{H}_{2} \mathrm{O}_{2}$-induced apoptosis $(10.5 \pm 3.7 \%, n=4$, $\left.{ }^{\star} P<0.01\right)$. Representative images of apoptotic cells identified by TUNEL staining showed reduced $\mathrm{H}_{2} \mathrm{O}_{2}$-induced apoptosis in Ad.TK-hEPCs compared with non-Adv-hEPCs and Ad.Null-hEPCs after $12 \mathrm{~h}$ (Figure $2 \mathrm{~b}$ ). Quantitative analysis verified these results, and the number of TUNEL-positive cells per high-powered field was quantified $(9.9 \pm 0.6$ in Ad.TK-hEPCs, $19.7 \pm 0.8$ in non-Adv-hEPCs, and $18.1 \pm 0.8$ in Ad.Null-hEPCs, ${ }^{\star} P<0.001$ vs non-Adv-hEPCs and Ad.Null-hEPCs, $n=3$; Figure $2 \mathrm{~d}$ ).

We also investigated the effect of TK on the Akt signaling pathway in hEPCs. Figure 2e shows that transduction of Ad.hTK in $\mathrm{H} 2 \mathrm{O} 2$-injured hEPCs resulted in increased phosphorylation of Akt and reduced cleaved caspase-3 levels.

The present study further investigated whether TK can inhibit caspase- 3 and caspase- 9 activation in $\mathrm{H} 2 \mathrm{O} 2$-treated hEPCs. After $\mathrm{H}_{2} \mathrm{O}_{2}$ treatment, caspase- 3 and caspase-9 activities were prominently increased, but TK gene overexpression significantly inhibited this $\left({ }^{\star} P<0.01\right.$ vs nonAdv-hEPCs and Ad.Null-hEPCs, $n=3$ ). Our results showed that TK protects hEPCs from oxidative stress-induced apoptosis via inhibition of activation of caspase-3 and caspase-9, and induction of Akt phosphorylation.

\section{TK-Modified hEPCs Upregulate VEGF Under Oxidative Stress}

To identify potential paracrine mechanisms responsible for the therapeutic effect of Ad.TK-hEPCs, levels of the angiogenic cytokine VEGF in the medium of Ad.TK-hEPCs under oxidative stress conditions for up to $24 \mathrm{~h}$ was significantly higher than that of Ad.Null-hEPCs $\left({ }^{*} P<0.01, n=3\right.$; Figure 2h).

\section{Effect of the hEPCs Conditioned Medium on $\mathrm{H}_{2} \mathrm{O}_{2}$-induced Neonatal Cardiomyocyte Apoptosis}

We hypothesized that the Ad.TK-hEPCs might release cytoprotective factors that prevent apoptosis in cardiomyocytes. To test this, neonatal rat ventricular cardiomyocytes were exposed to $\mathrm{H}_{2} \mathrm{O}_{2}$ for $12 \mathrm{~h}$ in the presence of either control or hypoxic conditioned medium from cultured Ad.Null- or Ad.TK-hEPCs. Our results showed that exposing neonatal rat ventricular cardiomyocytes to $12 \mathrm{~h}$ of $\mathrm{H}_{2} \mathrm{O}_{2}$ in hypoxiaconditioned medium from cultured Ad.TK-hEPCs results in a significant reduction in the number of apoptotic cardiomyocytes compared with similarly treated medium from cultured Ad.Null-hEPCs $(25.8 \pm 3 \%$ vs $39.8 \pm 5.6 \%, n=4$, $\left.{ }^{*} P<0.05\right)$. Furthermore, as compared with control medium, incubation in hypoxia-conditioned medium from cultured Ad.Null-hEPCs led to nearly a $33 \%$ decrease of the number of apoptotic cells $\left(51.5 \pm 6 \%,{ }^{\#} P<0.05\right.$; Figure $2 \mathrm{i}$ ).

\section{Correlation Between Cell Number and Fluorescence Intensity}

A small drop of DiD-labeled Ad.Null-hEPCs and Ad.hTK-hEPCs were examined by immunostaining on object slide to demonstrate the expression of TK. The expression of TK (brown) by adenovirus-mediated gene transfer was almost 100\% (Figure 3a). All cells labeled by DiD under fluorescence microscopy appeared in red, and staining efficiency was $100 \%$ (Figure 3a). DiD-labeled cells resuspended in agarose gel were transferred into 96-well plates at different cell concentrations, and fluorescence counts correlated linearly with cell number $\left(R^{2}=0.9977\right.$; Figure $3 \mathrm{~b}$ and $\mathrm{c}$ ).

\section{Ad.TK-hEPCs Reduce Ischemia-induced Cardiac Injury}

To evaluate the effect of Ad.TK-hEPCs in ischemia-induced cardiac injury, Ad.Null-hEPCs or Ad.TK-hEPCs were injected in the ischemic border area. Expression and localization of human TK in mouse heart were identified in the Ad.TK-hEPC-injected group, but not in the Ad.Null-hEPCinjected group (Figure 3d).

Data presented in Table 1 report that both Ad.Null-hEPCand Ad.TK-hEPC-treated MI mice have significantly smaller infarct size (38.8 $\pm 3.8 \%$ in Ad.Null-hEPC group; $24.7 \pm 6.3 \%$ in Ad.TK-hEPC group, $n=6)$ compared with mediumtreated MI mice $(51.7 \pm 4.4 \%, n=6)$, with Ad.TK-hEPCtreated mice showing smaller infarct size than those treated with Ad.Null-hEPCs $\left({ }^{\star} P<0.05\right.$, Figure $\left.3 e\right)$. Transthoracic echocardiographic examination was performed to evaluate cardiac function after MI. Ejection fraction (EF) was markedly reduced after MI, but was partially restored by EPC transplantation (Figure 3e; Table 1). Dramatic functional changes were detected in the Ad.TK-hEPC-treated mice as shown by echocardiography (Table 1). Ad.TK-hEPC-treated mice had significant improvements on function parameters (EF, FS, LVIDd, and LVIDs). However, there was only a positive trend toward improvement on FS between Ad.Null-hEPC-treated MI mice group and medium-treated group, and the difference did not reach statistical significance (Table 1). Systolic blood pressure was similar in all groups. MI tended to have lower SBP (Table 1).

MDA was measured in the infarcted myocardium to evaluate the role of oxidative stress after MI injury. MDAs were significantly elevated in the MI group compared with the sham group, and Ad.TK-hEPCs $(0.69 \pm 0.1 \mathrm{nmol} / \mathrm{mg}$ 
protein) significantly prevented the increase in cardiac MDA levels induced by MI damage $(1.3 \pm 0.1 \mathrm{nmol} / \mathrm{mg}$ protein in medium-treated group, $0.93 \pm 0.1 \mathrm{nmol} / \mathrm{mg}$ protein in
Ad.Null-hEPC-treated group, ${ }^{\star} P<0.05$ vs medium- and Ad.Null-hEPC-treated group. ${ }^{\#} P<0.05$ vs medium-treated group, Figure 3f).
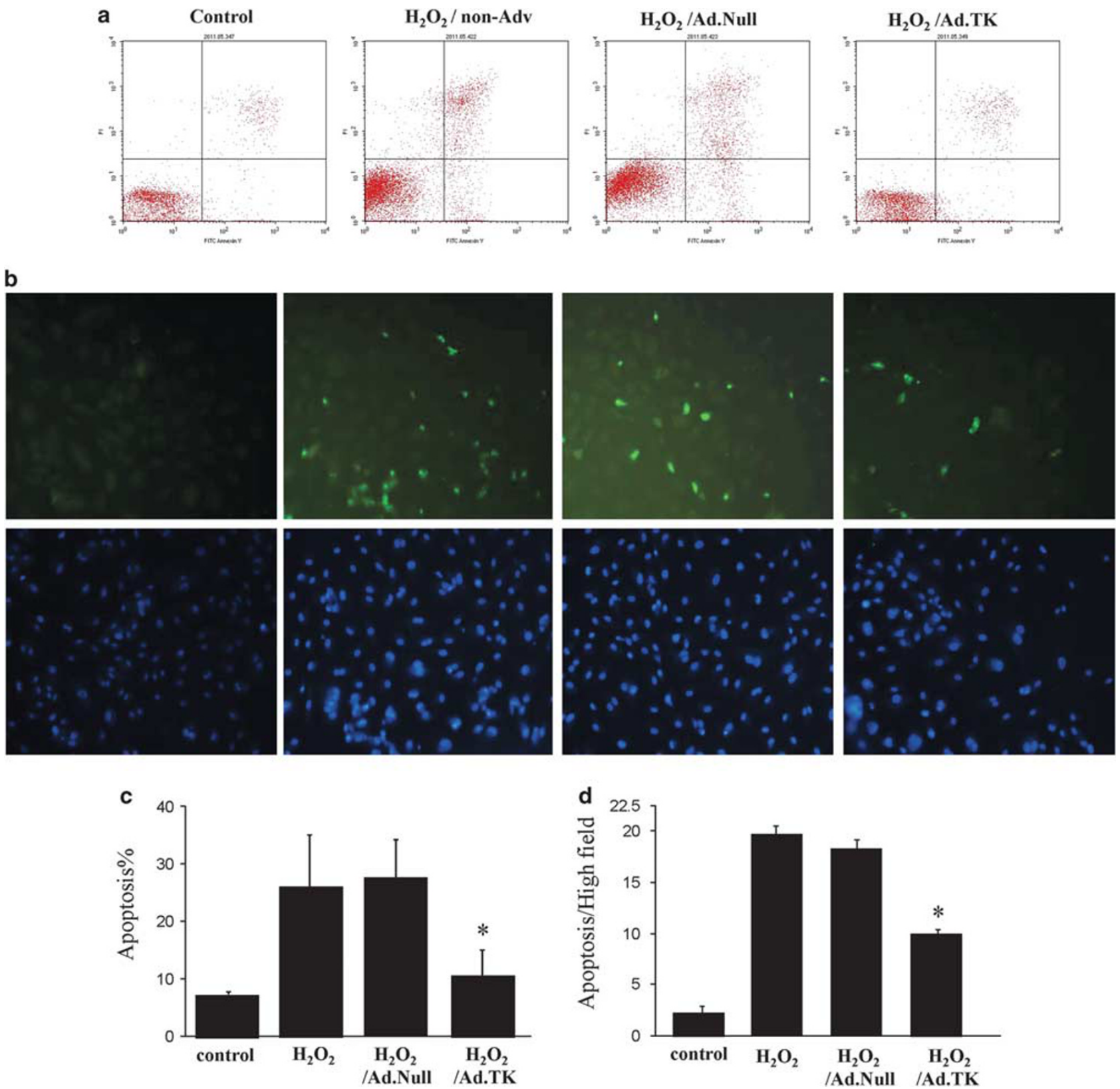

Figure 2 TK reduced EPC apoptosis induced by $\mathrm{H}_{2} \mathrm{O}_{2}$. (a) Representative flow-cytometric analysis of apoptosis showed that TK gene-transfer protects EPCs from $\mathrm{H}_{2} \mathrm{O}_{2}$-induced apoptosis. (b) In situ TUNEL assay was applied to examine cultured hEPC apoptosis (upper panel); lower panels showed staining of corresponding sections with the nuclear stain DAPI. (c) Quantitative analysis of EPC apoptosis by flow cytometry ${ }^{*} P<0.05$ vs other groups, $n=4)$. (d) Quantitative analysis of EPC apoptosis by TUNEL staining $\left({ }^{*} P<0.05\right.$ vs other groups, $\left.n=3\right)$. (e) Western blots for Akt and cleaved caspased-3. (f, g) Activation of caspase-3 and caspase-9 levels were significantly increased by exposure to $\mathrm{H}_{2} \mathrm{O}_{2}$, and TK gene transfer inhibited caspase-3 and caspase-9 activities. Results are the mean \pm s.e.m. from three independent experiments $\left({ }^{*} P<0.01 ; n=3\right)$. (h) VEGF secretion by Ad.TK-hEPCs under oxidative stress. After $24 \mathrm{~h}$ of incubation, conditioned medium from control and treated cells $(n=3)$ was subjected to VEGF ELISA assay. VEGF concentration values are mean \pm s.e.m. $\left({ }^{*} P<0.05\right)$. ELISA data are representative of three independent experiments. (i) Effect of the EPC conditioned medium on apoptosis of neonatal rat ventricular cardiomyocytes exposed to oxidative stress. Representative Hoechst 33324 staining images in neonatal rat ventricular cardiomyocytes treated with $0.2 \mathrm{mM} \mathrm{H}_{2} \mathrm{O}_{2}$ for $12 \mathrm{~h}$. Hoechst 33324 staining for nuclear morphology was performed to assess apoptotic cell death. Lower panel showed quantitative analysis of apoptosis levels using Hoechst 33324 staining in control medium, hypoxic Ad.NullhEPC-conditioned medium and hypoxic Ad.TK-hEPC-conditioned medium $12 \mathrm{~h}$ after $\mathrm{H}_{2} \mathrm{O}_{2} .{ }^{*} P<0.05$ vs control medium and hypoxic Ad.Null-hEPCconditioned medium; ${ }^{\#} P<0.05$ vs control medium, $n=4$ ). Original magnification is $\times 200$. 
e

g

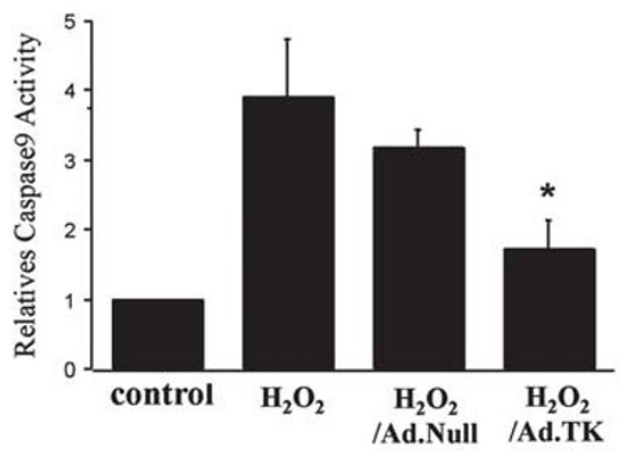

f

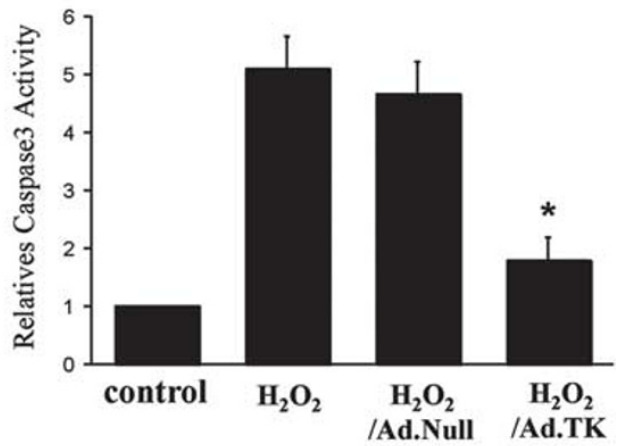

h

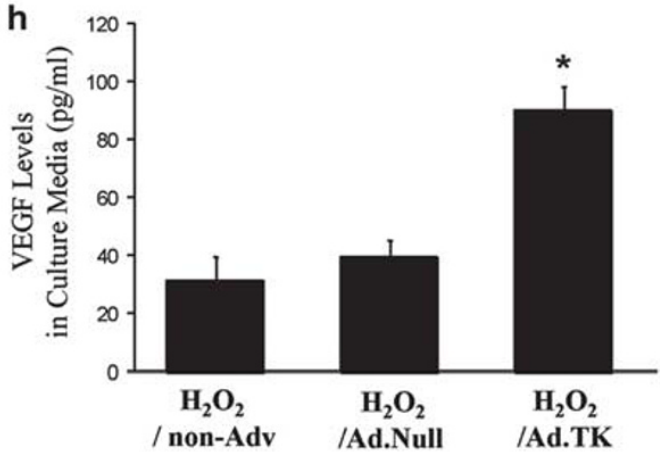

Hypoxia Ad.TK-hEPCs i

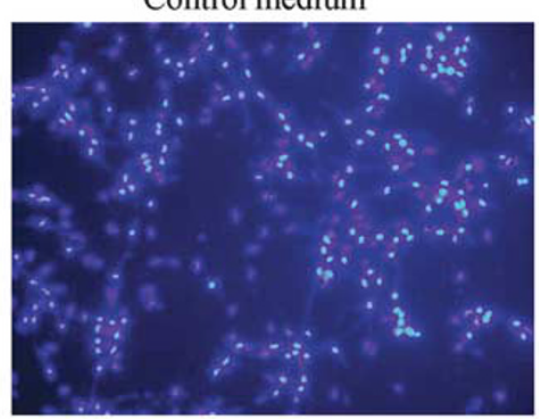

Hypoxia Ad.Null-hEPCs

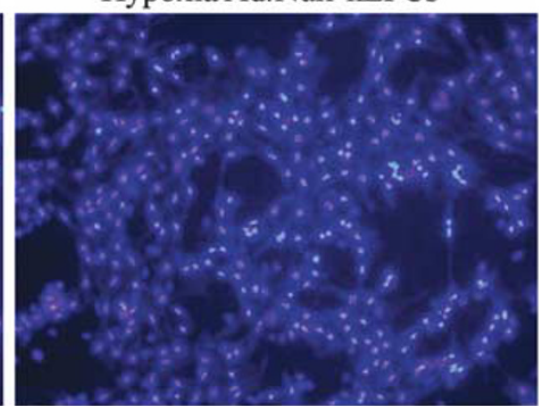

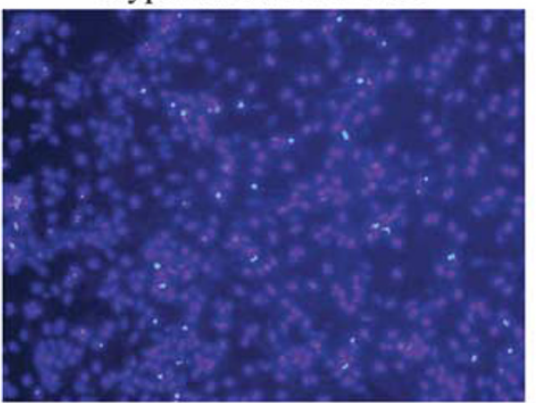

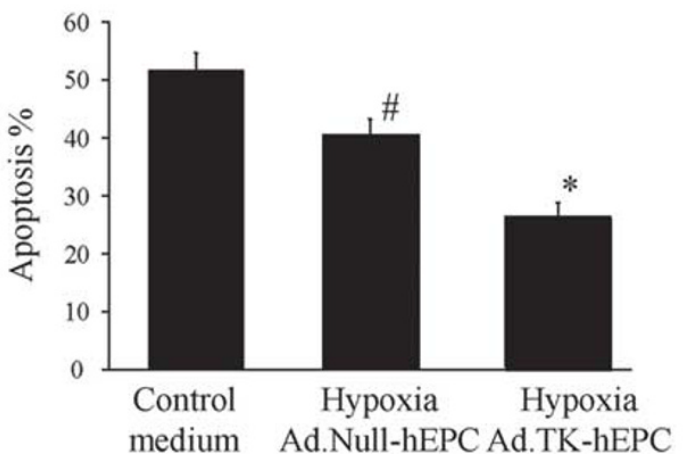

Figure 2 (Continued) 


\section{Transplanted Cell Survival Assessed by Optical Imaging} Because of the limited tissue penetration ability of DiD dye (emission wave length: $665 \mathrm{~nm}$ ), we were successful only in detecting the fate of transplanted cells ex vivo. The ex-vivo NIR fluorescent signals of transplanted DiD-labeled hEPCs were evaluated in ischemic hearts in Ad.Null-hEPC- and Ad.TK-hEPC-treated groups using optical imaging at days 2 and 7 after MI (Figure $4 \mathrm{a}$ and b). In addition to heart, there were slight fluorescent signals observed in other organs (lung, liver, spleen, and kidney) among two groups at days 2 and 7 . No fluorescent signals were observed in heart in mediumtreated MI mice at days 2 and days. Fluorescent signals of the heart in the Ad.TK-hEPC-treated group were remarkably increased compared with the Ad.Null-hEPC-treated group at two time points. Quantitative analysis of the fluorescent signals in explanted hearts among two EPC-transplanted groups showed that Ad.TK-hEPC injection resulted in the significantly higher fluorescent intensity compared with Ad.Null-hEPC injection from day 2 to day $7(0.0118 \pm 0.0012$ vs $0.0051 \pm 0.0017$ scaled counts/s for Ad.TK-hEPCs; $0.00648 \pm 0.00177$ vs $0.00233 \pm 0.00102$ scaled counts/s for Ad.Null-hEPCs, respectively, $n=3-4, P<0.001$ ). This means that the number of surviving cells in the Ad.TK-hEPC group was greater than in the Ad.Null-hEPC group following cell transplantation. From day 2 to day 7 , the fluorescent signals of explanted hearts in two EPC-transplanted groups significantly decreased.

\section{Histology and Immunohistochemistry}

Apoptotic cardiomyocytes were detected by TUNEL staining in the infarcted myocardium 2 days after MI. As shown in Figure 5a, there were more transplanted cells of DiD labeling a
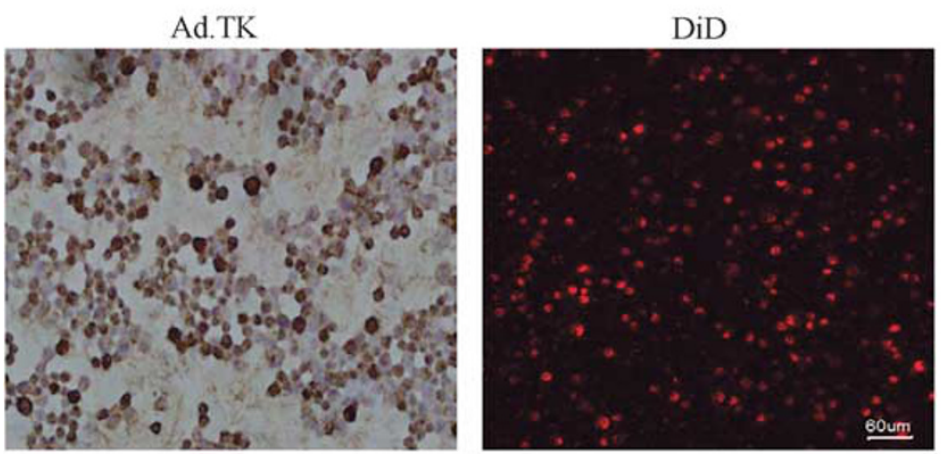

b

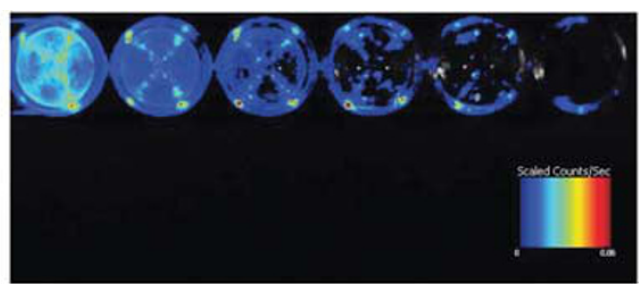

c

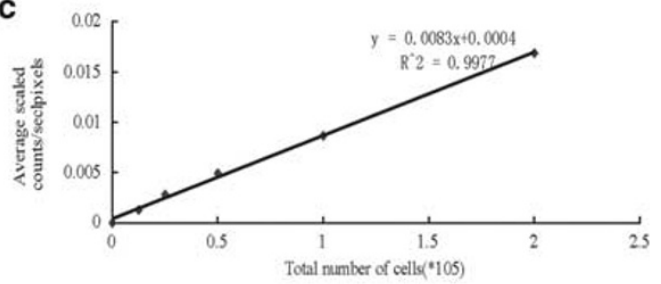

d TK

DiD
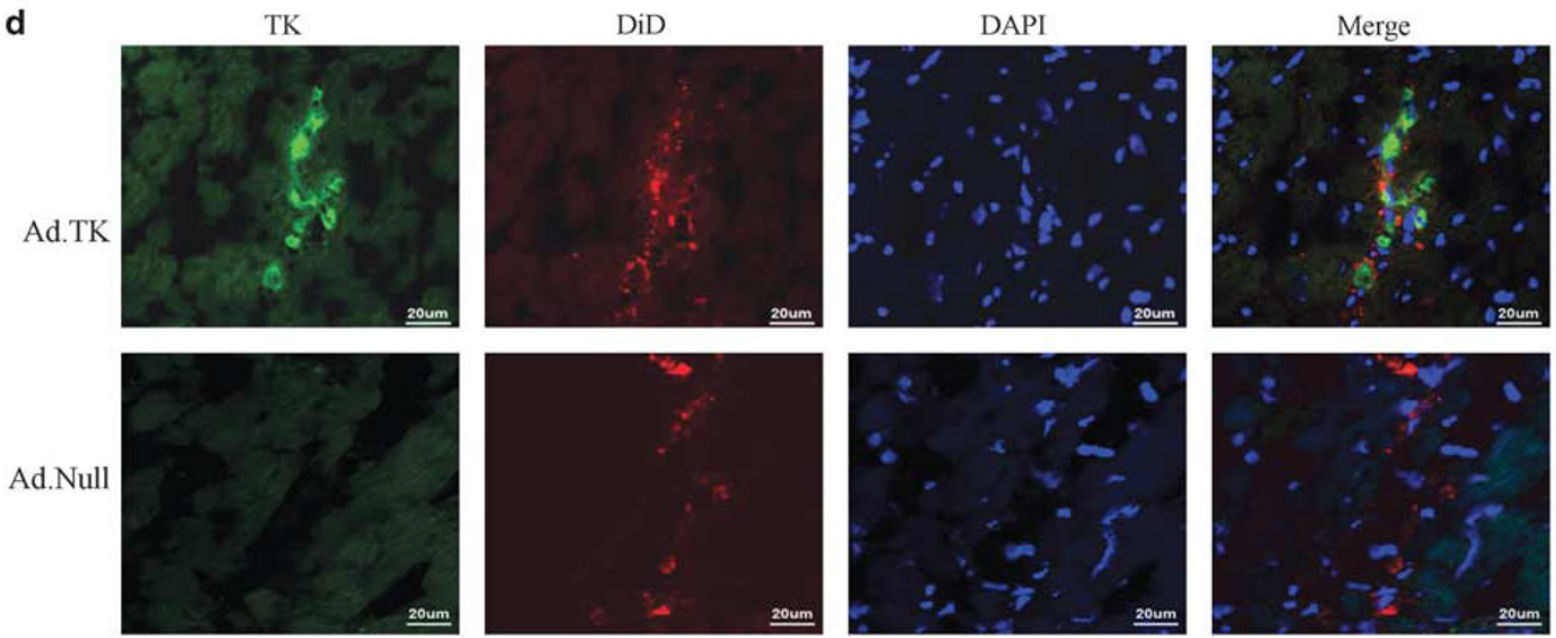
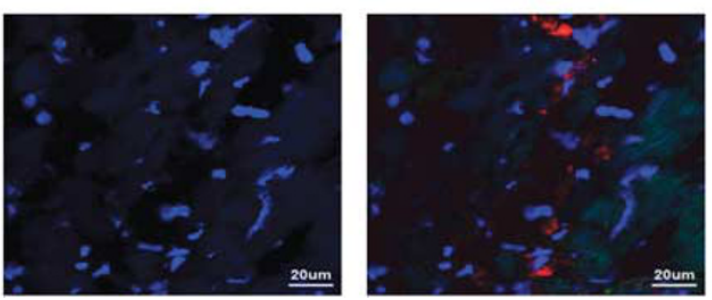

Figure 3 TK-modified hEPC transplantation reduced infarct size. (a) Expression of human TK in hEPCs after Ad.hTK transduction was confirmed by immunocytochemistry (left); the same view of DiD-labeled cells appeared in red under fluorescence microscopy $(\times 100$; excitation wavelength coverage 595-800 nm and emission coverage 660-680 nm). (b) Fluorescent images show increasing signal with increasing cell number in hEPCs. (c) Correlation plot shows robust correlation between signal and cell number $\left(R^{2}=0.9977\right)$. (d) Representative immunofluorescence photographs of DiD-labeled Ad.TKhEPC-transplanted group (red) and TK protein expression (green) in the infarcted region of mouse hearts 2 days after MI. Lower panel is DiD-labeled Ad.Null-hEPC-transplanted group (red). Original magnification is $\times 600$. (e) Representative Masson's trichrome staining. Original magnification is $\times 10$. (f) Echocardiographic measurements for determination of LV function from M-mode measurements. (g) MDA in the ischemic mouse heart at day 7 after MI. Values are expressed as mean \pm s.e.m. ( $n=6,{ }^{*} P<0.05$ vs Ad.Null-hEPC- and medium-treated group; ${ }^{\#} P<0.05$ vs medium-treated group). 

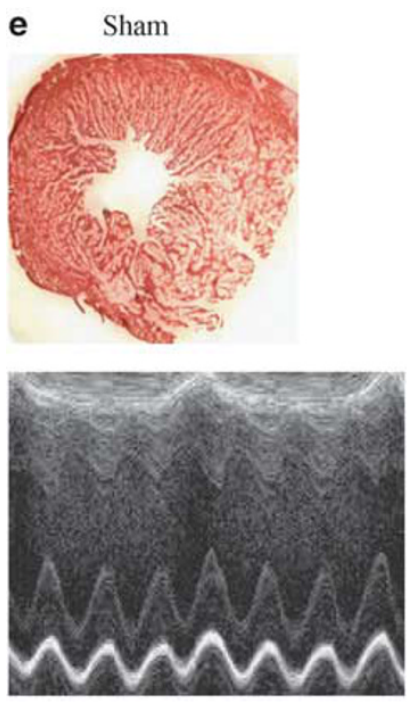
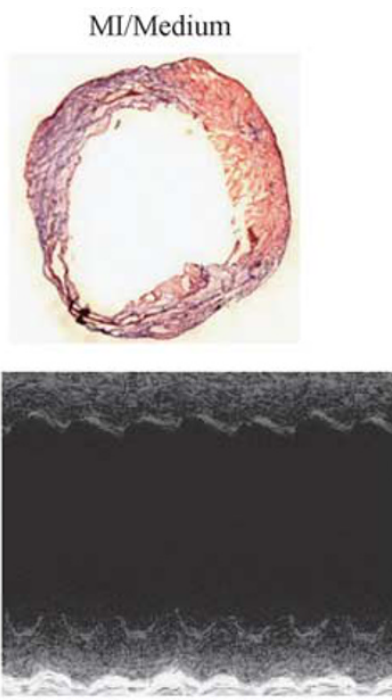
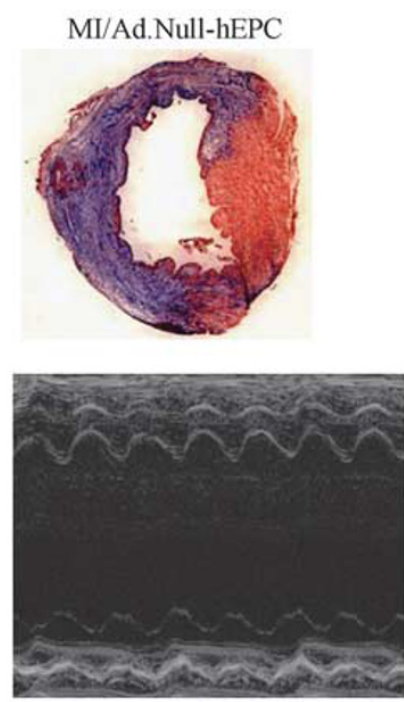
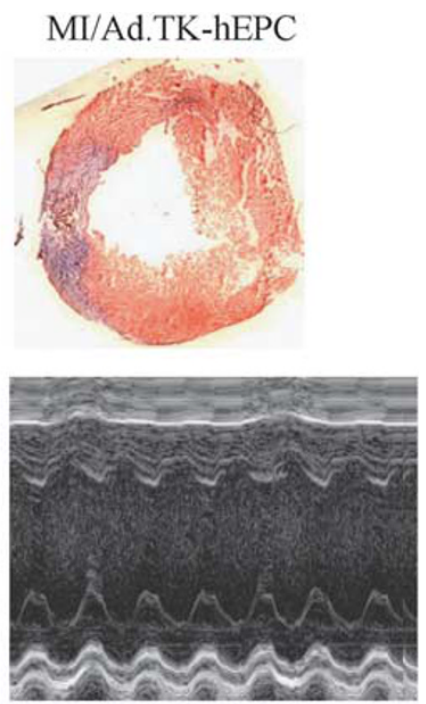

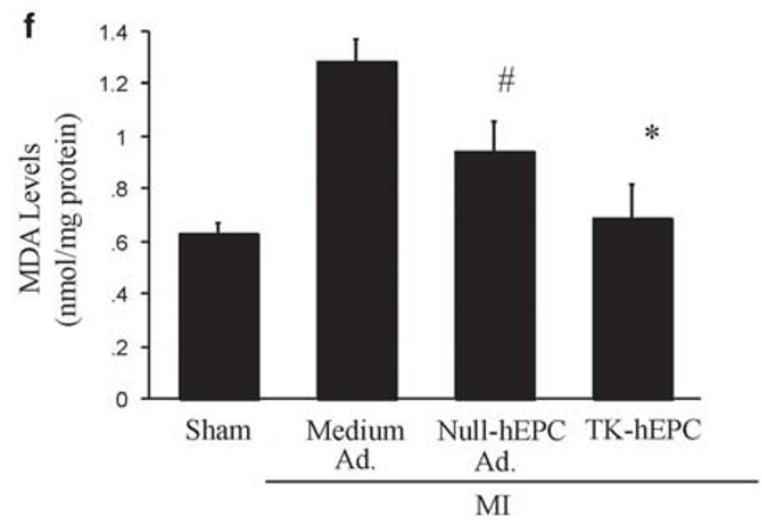

Figure 3 (Continued).

and less TUNEL-positive cardiomyocytes assessed by the confocal microscope in the Ad.TK-hEPC-injected group compared with the Ad.Null-hEPC-injected group at day 2 after MI. The ratio of TUNEL-positive cardiomyocytes to total number of cardiomyocytes in the Ad.TK-hEPC group was significantly reduced as compared with the Ad.NullhEPC- and medium-treated group $(22.6 \pm 2.3 \%$ vs $30.3 \pm 1.6 \%$ and $42.2 \pm 4.5 \%$, respectively, ${ }^{*} P<0.05, n=6$, Figure 5b). Ad.Null-hEPC-treated group showed decreases in apoptotic cells compared with medium-treated group ( $\left.{ }^{\#} P<0.05, n=6\right)$. Moreover, we examined the retention and survival of Ad.TK-hEPCs after their transplantation in the ischemic myocardium (2 days after MI) by assessing the number of DiD-labeled cells (red) in the myocardium. The Ad.hTK-hEPC-treated group showed significantly increased retention of transplanted EPCs in the myocardium after MI (Figure $5 \mathrm{~b}$ and $\mathrm{d}$ ).
Table 1 Effects of Kallikrein Modified hEPC on Cardiac Function 7 Days after MI

\begin{tabular}{lcccc}
\hline Variable & Sham & Ml/medium & Ml/Ad.Null-hEPC & Ml/Ad.TK-hEPC \\
\hline Infarct size (\%) & 0 & $51.7 \pm 4.4$ & $38.8 \pm 3.8^{\mathrm{a}}$ & $24.7 \pm 6.3^{\mathrm{b}}$ \\
EF (\%) & $76.4 \pm 8.3$ & $22.9 \pm 2.8$ & $32.1 \pm 3.3^{\mathrm{a}}$ & $52.1 \pm 3.3^{\mathrm{b}}$ \\
FS (\%) & $44.3 \pm 7.8$ & $10.0 \pm 0.7$ & $15.1 \pm 2.0$ & $25.8 \pm 2.9^{\mathrm{b}}$ \\
LVIDd (mm) & $3.2 \pm 0.1$ & $5.1 \pm 0.3$ & $4.1 \pm 0.5^{\mathrm{a}}$ & $3.4 \pm 0.2^{\mathrm{b}}$ \\
LVIDs (mm) & $1.74 \pm 0.2$ & $4.6 \pm 0.3$ & $3.5 \pm 0.5^{\mathrm{a}}$ & $2.5 \pm 0.2^{\mathrm{b}}$ \\
SBP (mm Hg) & $105 . \pm 8.3$ & $96 \pm 5.5$ & $97.9 \pm 7.7$ & $100.9 \pm 5.4$
\end{tabular}

Abbreviations: EF, ejection fraction; FS, fractional shortening; LVIDd, left ventricular inner diastolic diameter; LVIDs, left ventricular inner systolic diameter; MI, myocardial infarction; SBP, systolic blood pressure.

Values are mean \pm s.e.m.; $n=6$ in each group.

${ }^{\mathrm{a}} \mathrm{P}<0.05$ vs $\mathrm{Ml} /$ medium.

${ }^{\mathrm{b}} \mathrm{P}<0.05$ vs $\mathrm{Ml} /$ medium and Ml/Ad.Null-hEPC. 


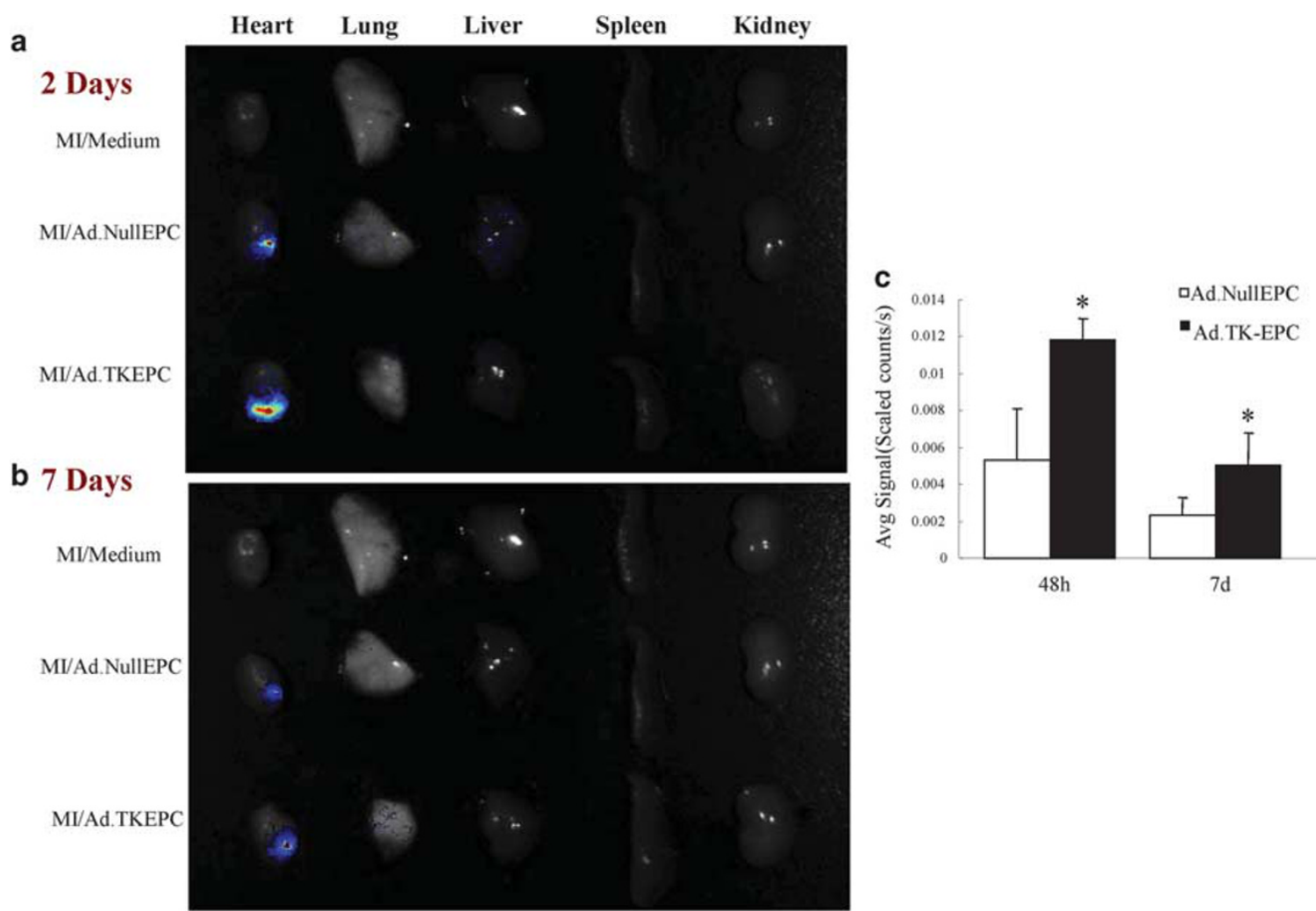

Figure 4 Ex-vivo optical imaging study. (a, b) Representative NIR fluorescent images in explanted organs at days 2 or 7 following implantation of DiDlabeled hEPCs into the ischemic myocardium of nude mice. Bars represent maximum radiance. (a: 2 days after cell delivery; b: 7 days after cell delivery). (c) Quantitative analysis of NIR fluorescent signals in explanted hearts among each group at two time points. All values are expressed as mean \pm s.e.m. ( $n=3-4, * P<0.01$ vs control group).

Capillary density in the peri-infarct area, as evaluated by CD31 immunohistochemistry, was significantly greater in Ad.TK-hEPC-treated MI mice than what was detected in medium- or Ad.Null-hEPC-injected MI mice $(179.3 \pm 23.4$ vs $74 \pm 10.7$ and $127.0 \pm 24.7$ capillaries $/ \mathrm{mm}^{2}$, respectively, ${ }^{\star} P<0.05, n=6$, Figure 6a). Consistent with our functional data, modest increases in capillary density were observed in Ad.Null-hEPC-injected group compared with controls, who displayed no obvious angiogenesis ( ${ }^{\#} P<0.05$, Figure 6$)$. In sham group, the density of capillary was $1704.4 \pm 290 / \mathrm{mm}^{2}$. Moreover, small arterioles were clearly visible in the tissue sections stained with an antibody against $\alpha$-smooth muscle actin (Figure 6b). Quantitative analysis showed that arteriolar density was markedly increased in the Ad.TK-hEPC-injected group compared with medium- or Ad.Null-hEPC-injected MI mice $\left(121.3 \pm 7.9\right.$ vs $47.3 \pm 10.9$ and $89.7 \pm 18.4 / \mathrm{mm}^{2}$, respectively, ${ }^{*} P<0.05, n=6$, Figure $6 \mathrm{~d}$ ); there was a significant increase in arteriole density in Ad.Null-hEPC-treated mice $\left(89.7 \pm 18.4 / \mathrm{mm}^{2},{ }^{\#} P<0.05\right.$ vs medium-treated mice, $n=6)$ when compared with medium-treated mice. The density of arteriole density in sham was $72.8 \pm 11.1 / \mathrm{mm}^{2}$.

As shown in Figure 6e, DiD-labeled implanted cells were incorporated into capillaries and arterioles and were assessed by a confocal microscope in high power field at the border zone of the ischemic myocardium in the Ad.TK-hEPC group. This demonstrates that transplanted cells may directly participate in angiogenesis.

\section{DISCUSSION}

This study demonstrates that a combined EPC and kallikrein gene approach provides advanced benefits in healing injured heart in a setting of acute permanent coronary artery ligation.

Stem cells have been proposed as a promising cell source for the regeneration and restoration of infarcted heart function. However, poor survival of implanted cells hampered the therapeutic efficacy. In this study, overexpression of TK inhibited oxidative stress-induced apoptosis in cultured human umbilical cord blood-derived EPCs. Expression of human TK in mouse heart after Ad.TK-hEPCs implantation exhibited enhanced protection against cardiac injury by inhibiting apoptosis and promoting angiogenesis. In-vivo transplantation of Ad.TK-hEPCs enhanced the survival rate of EPCs in the LAD ligation mice. Moreover, Ad.TK-hEPC implantation attenuated infarction size and restored LV function, which may have attributed to the enhanced antiapoptotic properties of the TK-modified EPCs. Our results agree with the earlier findings that overexpression of TK 
through gene delivery or protein infusion in the heart reduced infarct size and improved recovery of cardiac function after ischemia and/or reperfusion injury with antioxidative, antiapoptotic, antiinflammatory, and angiogenic effects. ${ }^{9,10,15}$
Human cord blood cells are rich in stem and progenitor cells. Research has shown that significantly larger numbers of EPCs are found in cord blood compared with peripheral blood. ${ }^{15,16}$ In addition, transplantation of EPCs could successfully promote neovascularization in animal a
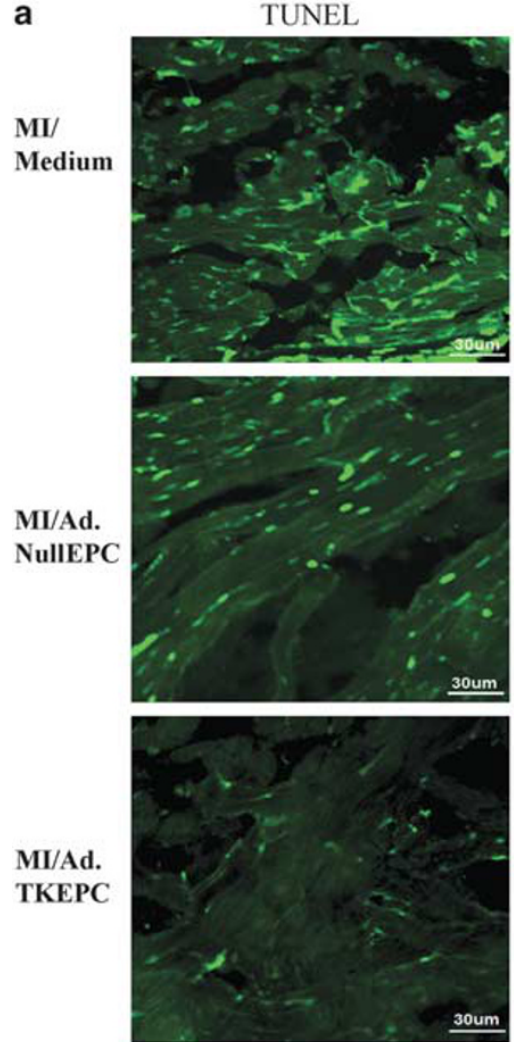

b



40um

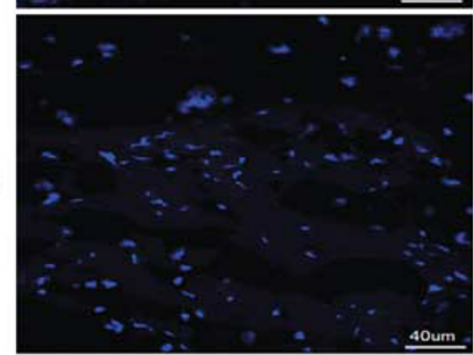

DID

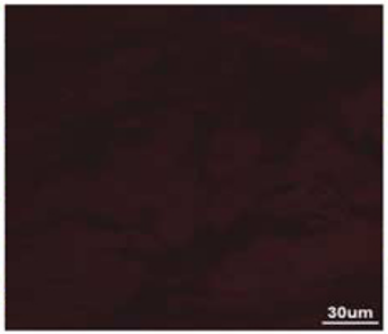

30um
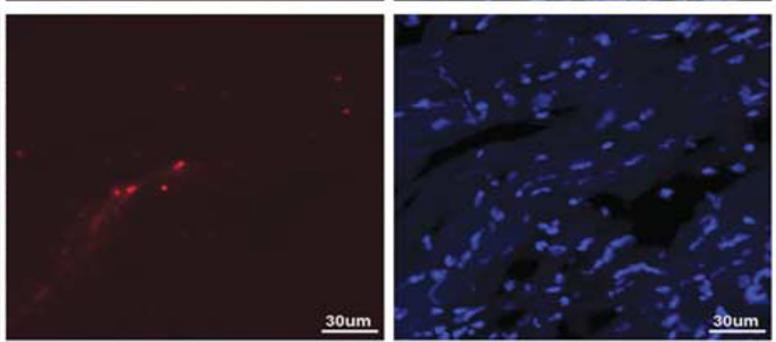

DAPI
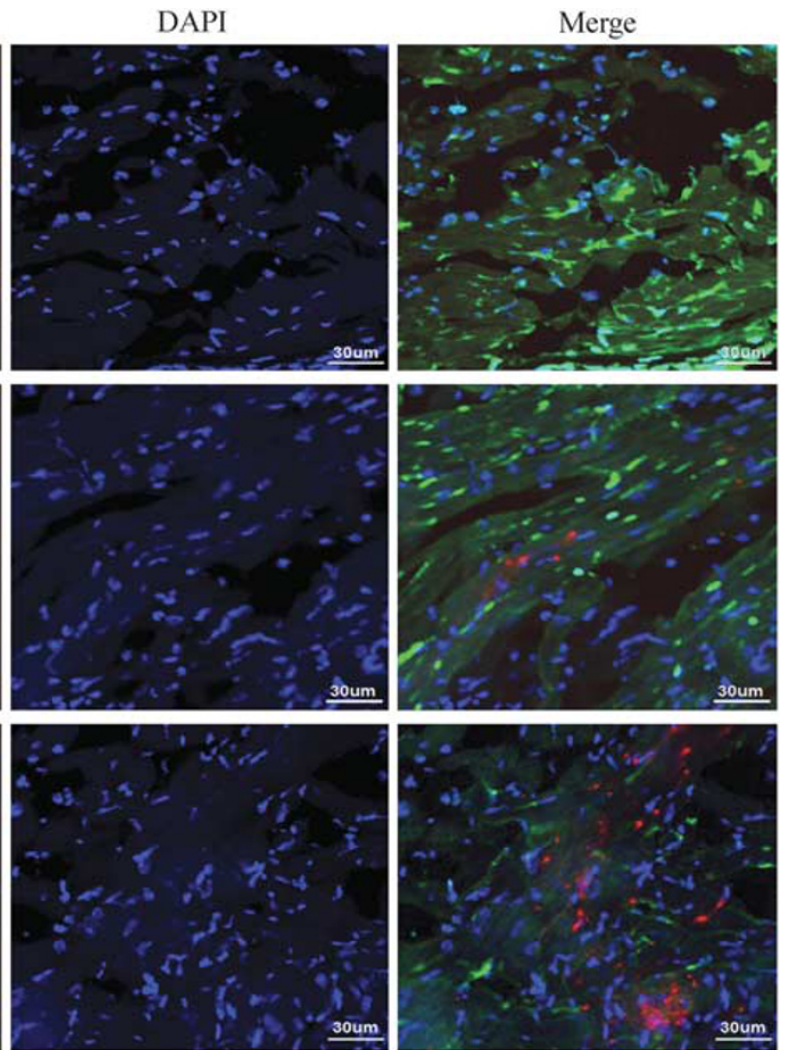

DID
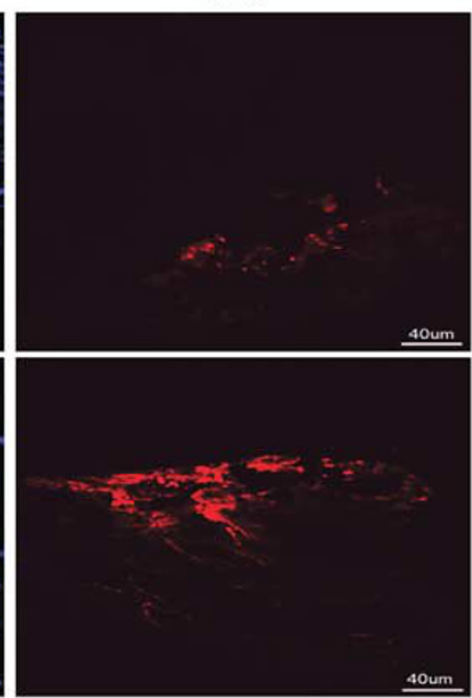

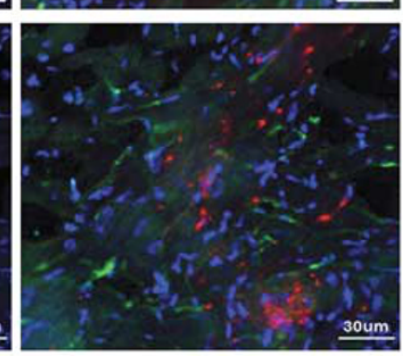

Merge

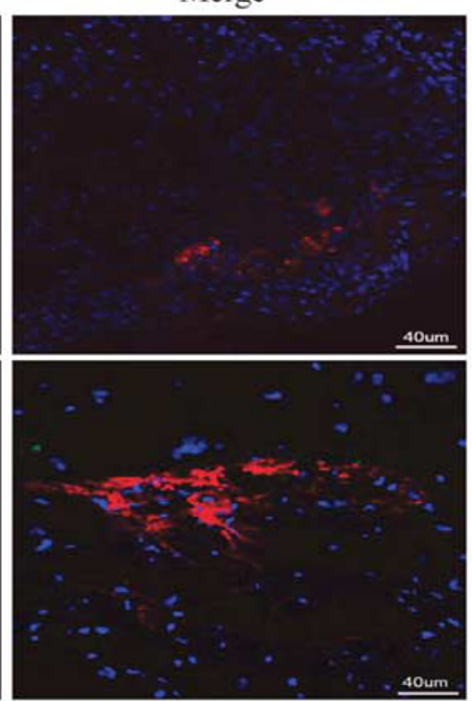

Figure 5 Confocal immunofluorescent images of DiD-labeled hEPCs and TUNEL-positive cardiomyocytes. (a) TUNEL-positive cardiomyocytes (green) and NIR fluorescent signals (red) were co-observed in the myocardium at day 2; counterstaining was performed with DAPI (blue). Original magnification is $\times 600$. (b) Representative micrographs show DiD-positive cells in mice heart at day 7 after Ml (red, $\times 400$ magnification). Nuclei were counterstained with DAPI (blue). (c) Quantitative analysis of apoptotic cardiomyocytes expressed as percentage of TUNEL-positive nuclei in cardiomyocytes. TUNELpositive noncardiomyocytes were excluded ( $n=6,{ }^{*} P<0.05$ vs Ad.Null-hEPCs- and medium-treated group; ${ }^{\#} P<0.05$ vs medium-treated group). (d) Quantitative analysis of DiD-positive cells in mice heart at day 2 after MI ( $n=6,{ }^{*} P<0.05$ vs Ad.Null-hEPC-treated group). 

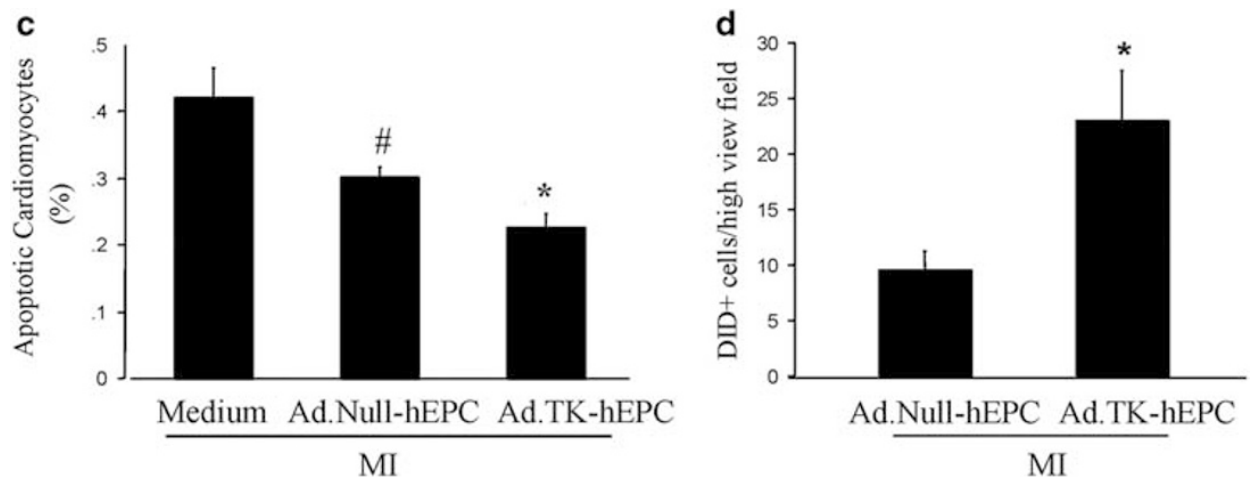

Figure 5 (Continued).

experiments and clinical patients. ${ }^{17,18}$ However, the background risk factors in patients that may reduce the number and functional activity of EPCs represent major limitations for the success of EPC transplantation. ${ }^{19}$ The use of cord blood may make stem cell transplants available more quickly for patients with severe cases of MI who need the cells as soon as possible. ${ }^{20}$ In this study, we isolated EPCs from human cord blood using a common methodology. These cultured cells exhibited increased expression of EPC markers (CD34 and KDR) at passage one, and consequently were considered as EPCs. In the in-vivo study, $5 \times 10^{5}$ EPCs were transplanted through intramyocardial injection in nude mice after MI, and the EPC-transplanted group show promising results. EPC transplantation was associated with smaller infarcts compared with medium injection. Our results confirmed that cord blood is a better source for EPCs. On the other hand, compared with EPC control group, TKmodified EPCs showed the best improvement.

Several studies from our group and others have confirmed that the KKS inhibits intramyocardial inflammation and oxidative stress in experimental diabetic cardiomyopathy ${ }^{21}$ and has an important role in cardioprotection during ischemic injury. ${ }^{9,10,11,15}$ Urbich et al. ${ }^{22}$ have demonstrated that reactive oxygen species, which are known to be increased in patients with coronary heart disease or diabetes, profoundly reduce the number of viable EPCs by inducing apoptosis. In this study, $\mathrm{H}_{2} \mathrm{O}_{2}$ was used to mimic oxidative stress in vitro, we found that TK inhibited hEPC apoptosis in conjunction with increased Akt phosphorylation and reduced caspase- 3 and caspase- 9 activation. We postulated that the antiapoptotic effect of TK against ischemic injure may be mediated by a positive feedback interaction with the phosphatidylinositol 3-kinase (PI3K)-Akt survival pathway. Moreover, compared with the medium-treated MI animals and Ad.Null-hEPC-treated group, Ad.TK-hEPC transplantation significantly decreased oxidative stress in heart tissue. Implantation of genetically modified hEPCs expressing human TK prevented cardiomyocyte apoptosis in the initial phase after acute ischemic injury. Our study documented significant retention and survival of Ad.TKhEPCs in ischemic heart that was associated with inhibition of ventricular cardiomyocyte apoptosis, and almost restored an impaired systolic function and diastolic cardiac function. These combined results from in-vivo and in-vitro studies suggest that Ad.TK-hEPCs protect against ROS-induced cellular apoptosis. Our data also indicate that the number of viable transplanted cells is an important factor for preservation of cardiac function. This is consistent with our previous results, indicating that implantation of kallikreinmodified MSCs in the kidney provides advanced benefits in protection against ischemia-induced kidney injury by suppression of apoptosis and inflammation. ${ }^{23}$

NIR fluorescent dye molecule provides highly sensitive and temporal resolution for in-vitro cell labeling and in-vivo cell tracking with optical imaging. ${ }^{24}$ Our study showed that EPClabeling efficiency by NIR fluorescent dye DiD was almost $100 \%$, and a positive good correlation between the number of labeled cells and fluorescence intensity measurement was observed. In addition, cellular engraftment was determined in explanted organs at days 2 and 7 by measuring ex-vivo optical signals. We describe a simple way to monitor the survival of transplanted stem cells. The methods consistently showed that the average signals of heart with $\mathrm{DiD}$ in the Ad.TK-hEPC-transplanted group were stronger than Ad.Null-hEPC-transplanted group at two time points. Higher survival of EPCs was also found in the Ad.TKhEPC-transplanted group compared with Ad.Null-hEPCtransplanted group by pathological detections. Moreover, TUNEL staining showed decreased TUNEL-positive cells in the Ad.TK-hEPC-transplanted group. Increased retention of transplanted Ad.TK-hEPCs in the myocardium may decrease apoptotic rates of cardiomyocytes. In this study, although NIR dye labeling showed good results, to further evaluate the duration of transplanted cell survival, integration and proliferation in target organs, multimodality imaging techniques combined with the relative advantages of MRI, 

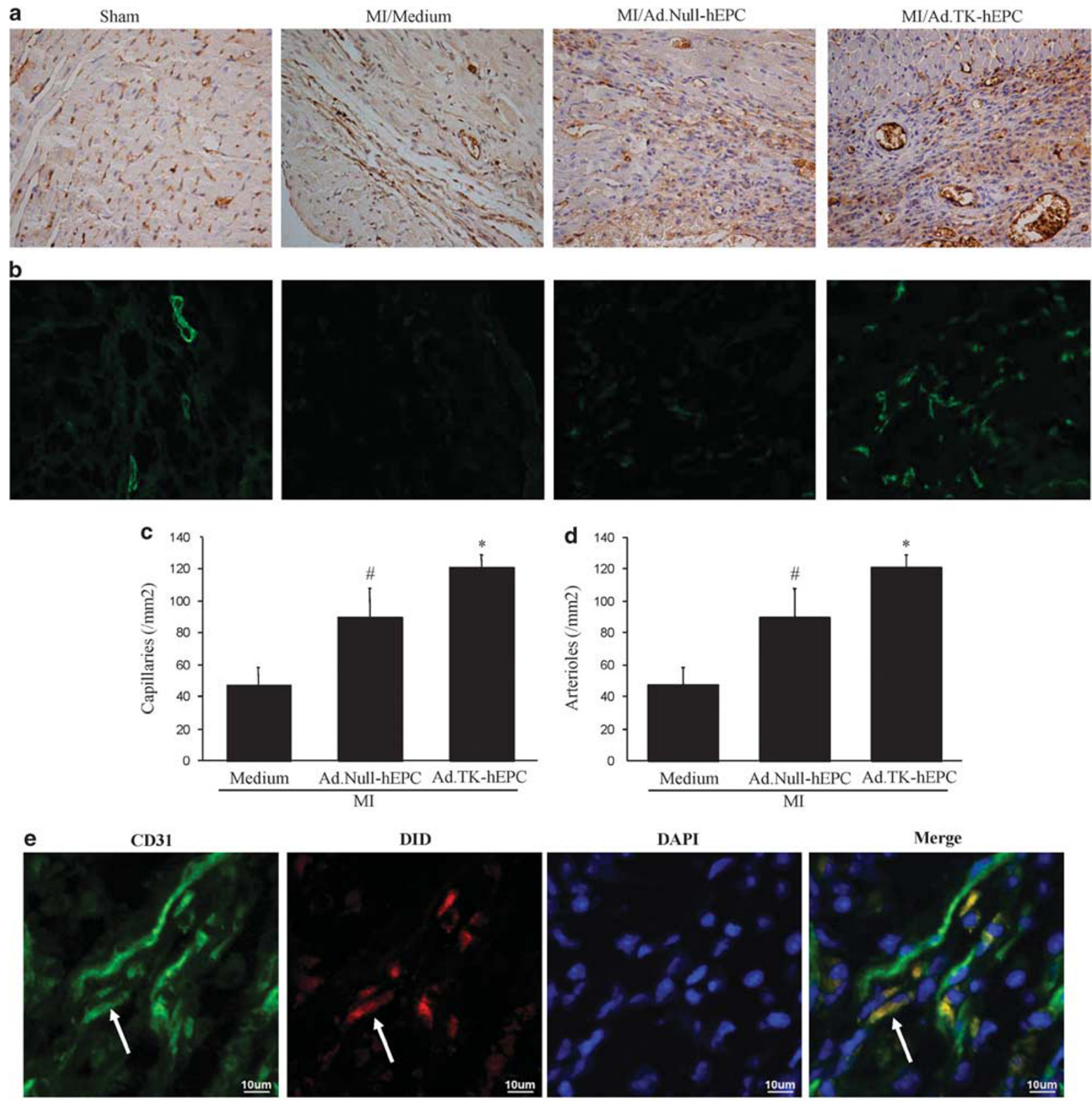

$\alpha$ - SMA
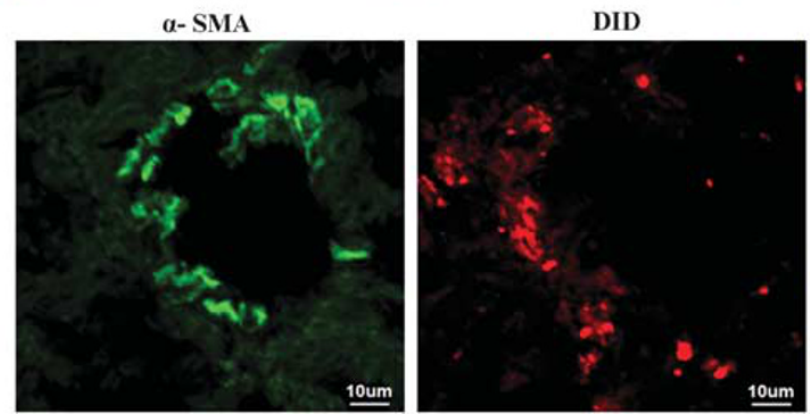
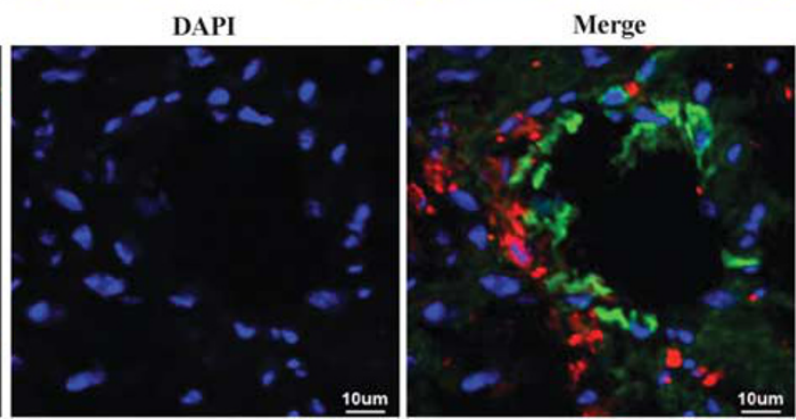

Figure 6 TK-modified hEPC transplantation increased capillary density and arteriolar density 7 days after MI. Representative photographs of immunostaining using (a) CD31 to identify capillaries and (b) $\alpha$-SMA to identify arterioles. Original magnification is $\times 200$. Quantitative analysis of (c) capillary density and (d) arteriolar density in the peri-infarct myocardium ( $n=6,{ }^{*} P<0.05$ vs Ad.Null-hEPC- and medium-treated group; ${ }^{*} P<0.05$ vs medium-treated group). (e) High power field of DiD-labeled implanted hEPCs (red) and immunofluorescent staining of CD31 or $\alpha$-SMA (green) at the border zone of the ischemic myocardium; nuclei were counterstained with DAPI (blue). Original magnification is $\times 1200$. Transplanted DiD-labeled hEPCs were clearly incorporated into $\mathrm{CD}_{3} 1^{+}$capillaries and $\alpha$-SMC ${ }^{+}$small arteries in the Ad.TK-hEPC group. 
PET, SPECT, optical imaging, and ultrasound, ${ }^{25}$ will provide complementary and reliable information to assess stem cell engraftment in the recipient tissue in our future study.

Our current research, together with many other studies, demonstrated that EPC implantation could induce therapeutic angiogenesis in infarcted heart. ${ }^{3,26}$ It was reported that cell transplantation using VEGF-expressing MSCs could enhance the cardioprotective effects of MSCs, followed by angiogenesis effects in salvaging host myocardium. ${ }^{27}$ In this study, Ad.TK-hEPC transplantation led to increases in both angiogenesis and arteriogenesis at the border zone of the ischemic myocardium. In-vitro data confirmed a significant increase of VEGF secretion by Ad.TK-hEPCs, which may participate in angiogenesis. ${ }^{28}$ Our data demonstrated the cytoprotective effect of hypoxic conditioned medium from cultured Ad.TK-hEPCs on isolated neonatal rat ventricular cardiomyocytes exposed to oxidative stress in vitro. An alternative explanation of our results is that there were significantly more DiD-positive hEPCs incorporated into the vasculature in the Ad.TK-hEPC group than in the control group. Our evidence supports the theory that using a genetic modification approach may further enhance the cytoprotective effect and restore EPC angiogenesis effect.

However, the mechanism as to how TK overexpression results in VEGF secretion was not explored. It is clear that the process of vasculogenesis requires an elaborate cascade of signaling events capable of mobilizing, homing, and retaining EPCs. ${ }^{29}$ In addition, prior research has shown that the PI3K/ Akt-dependent hypoxia-inducible factor 1 (HIF-1) activation pathway increases expression of the angiogenic genes VEGF, Flt-1, and MMP-2 in ischemic tissue and BM-derived progenitor cells. ${ }^{30}$ Although there are no reports indicating a significant correlation between hypoxia and TK levels in vivo, the present study shows that TK overexpression induces VEGF secretion in vitro. However, to fully understand this, the molecular mechanisms will need to be explored. In our future studies, we plan to examine whether TK is able to enhance HIF-1 $\alpha /$ VEGF/MMP-2 activation through activation of the B2 receptor-mediated PI3K/Akt signaling pathway.

In summary, we have confirmed that genetic modification of EPCs with the human TK gene resulted in high levels of Akt phosphorylation and VEGF expression in response to oxidative conditions. TK-modified EPCs enhanced the survival of engrafted EPCs in the heart after acute MI. The transplantation of TK-modified EPCs improved LV function. Therefore, transplantation of TK gene-engineered EPCs may provide a novel and effective approach in the treatment of acute MI.

\section{ACKNOWLEDGEMENTS}

We would like to acknowledge grant support from the National Natural Science Foundation of China (30871071 and 81070085 to YY) and the National Institute of Health (HL29397).

\section{DISCLOSURE/CONFLICT OF INTEREST}

The authors declare no conflict of interest.

1. Schuh A, Liehn EA, Sasse A, et al. Transplantation of endothelial progenitor cells improves neovascularization and left ventricular function after myocardial infarction in a rat model. Basic Res Cardiol 2008;103:69-77.

2. Leone $A M$, Valgimigli $M$, Giannico $M B$, et al. From bone marrow to the arterial wall: the ongoing tale of endothelial progenitor cells. Eur Heart J 2009;30:890-899.

3. Jujo K, li M, Losordo DW. Endothelial progenitor cells in neovascularization of infarcted myocardium. J Mol Cell Cardiol 2008;45:530-544.

4. Wu JC, Chen IY, Sundaresan G, et al. Molecular imaging of cardiac cell transplantation in living animals using optical bioluminescence and positron emission tomography. Circulation 2003;108:1302-1305.

5. Mangi AA, Noiseux N, Kong D, et al. Mesenchymal stem cells modified with Akt prevent remodeling and restore performance of infarcted hearts. Nat Med 2003;9:1195-1201.

6. Silva Jr J-A, Araujo RC, Baltatu O, et al. Reduced cardiac hypertrophy and altered blood pressure control in transgenic rats with the human tissue kallikrein gene. FASEB J 2000;14:1858-1860.

7. Nolly HL, Saed G, Scicli G, et al. The kallikrein-kinin system in cardiac tissue. Agents Actions Suppl 1992;38:62-72.

8. Kränkel N, Katare RG, Siragusa M, et al. Role of kinin B2 receptor signaling in the recruitment of circulating progenitor cells with neovascularization potential. Circ Res 2008;103:1335-1343.

9. Yao YY, Yin H, Shen B, et al. Tissue kallikrein promotes neovascularization and improves cardiac function by the Akt-glycogen synthase kinase-3\{beta\} pathway. Cardiovasc Res 2008;80:354-364.

10. Yin $\mathrm{H}$, Chao L, Chao J. Kallikrein/kinin protects against myocardial apoptosis after ischemia/reperfusion via Akt-glycogen synthase kinase-3 and Akt-Bad.14-3-3 signaling pathways. J Biol Chem 2005;280:8022-8030.

11. Yao $Y$, Sheng $Z$, Li $Y$, et al. Tissue kallikrein promotes cardiac neovascularization by enhancing endothelial progenitor cell functional capacity. Hum Gene Ther 2012;23:859-870.

12. Yoshida $\mathrm{H}$, Zhang JJ, Chao L, et al. Kallikrein gene delivery attenuates myocardial infarction and apoptosis after myocardial ischemia and reperfusion. Hypertension 2000;35:25-31.

13. Aikawa R, Komuro I, Yamazaki T, et al. Oxidative stress activates extracellular signal-regulated kinases through src and ras in cultured cardiac myocytes of neonatal rats. J Clin Invest 1997;100:1813-1821.

14. Krege JH, Hodgin JB, Hagaman JR, et al. A noninvasive computerized tail-cuff system for measuring blood pressure in mice. Hypertension 1995;25:1111-1115.

15. Yao YY, Yin $\mathrm{H}$, Shen B, et al. Kallikrein infusion minimizes myocardial infarction and inflammation through suppression of oxidative stress and mitogen-activated protein kinase signaling pathway. Regulatory Peptides 2007:5:12-20.

16. Peichev M, Naiyer AJ, Pereira D, et al. Expression of VEGFR-2 and AC133 by circulating human $\mathrm{CD} 34(+)$ cells identifies a population of functional endothelial precursors. Blood 2000;95:952-958.

17. Iwakura A, Shastry S, Luedemann C, et al. Estradiol enhances recovery after myocardial infarction by augmenting incorporation of bone marrow-derived endothelial progenitor cells into sites of ischemia-induced neovascularization via endothelial nitric oxide synthase-mediated activation of matrix metalloproteinase-9. Circulation 2006;113:1605-1614.

18. Kawamoto A, Katayama M, Handa N, et al. Intramuscular transplantation of G-CSF-mobilized CD34( + ) cells in patients with critical limb ischemia: a phase I/lla, multicenter, single-blinded, doseescalation clinical trial. Stem Cells 2009;27:2857-2864.

19. Asahara T, Kawamoto A, Masuda H. Concise review: circulating endothelial progenitor cells for vascular medicine. Stem Cells 2011;29:1650-1655.

20. Leor J, Guetta E, Feinberg MS, et al. Human umbilical cord bloodderived CD133 + cells enhance function and repair of the infarcted myocardium. Stem Cells 2006;24:772-780.

21. Tschöpe C, Walther T, Escher F, et al. Transgenic activation of the kallikrein-kinin system inhibits intramyocardial inflammation, endothelial dysfunction and oxidative stress in experimental diabetic cardiomyopathy. FASEB J 2005;19:2057-2059. 
22. Urbich C, Knau A, Fichtlscherer S, et al. FOXO-dependent expression of the proapoptotic protein Bim: pivotal role for apoptosis signaling in endothelial progenitor cells. FASEB J 2005;19:974-976.

23. Hagiwara M, Shen B, Chao L, et al. Kallikrein-modified mesenchymal stem cell implantation provides enhanced protection against acute ischemic kidney injury by inhibiting apoptosis and inflammation. Hum Gene Ther 2008;19:807-819.

24. Ferrari A, Hannouche $D$, Oudina $K$, et al. In vivo tracking of bone marrow fibroblasts with fluorescent carbocyanine dye. J Biomed Mater Res 2001;56:361-367.

25. Lau JF, Anderson SA, Adler E, et al. Imaging approaches for the study of cell-based cardiac therapies. Nat Rev Cardiol 2010;7:97-105.

26. Kawamoto A, Gwon HC, Iwaguro $\mathrm{H}$, et al. Therapeutic potential of ex vivo expanded endothelial progenitor cells for myocardial ischemia. Circulation 2001;103:634-637.
27. Matsumoto $\mathrm{R}$, Omura $\mathrm{T}$, Yoshiyama $\mathrm{M}$, et al. Vascular endothelial growth factor-expressing mesenchymal stem cell transplantation for the treatment of acute myocardial infarction. Arterioscler Thromb Vasc Biol 2005;25:1168-1173.

28. Uemura $R, X u M, A h m a d ~ N$, et al. Bone marrow stem cells prevent left ventricular remodeling of ischemic heart through paracrine signaling. Circ Res 2006;98:1414-1421.

29. Chang El, Loh SA, Ceradini DJ, et al. Age decreases endothelial progenitor cell recruitment through decreases in hypoxia-inducible factor 1alpha stabilization during ischemia. Circulation 2007;116: 2818-2829.

30. Cheng XW, Kuzuya $M$, Kim W, et al. Exercise training stimulates ischemia-induced neovascularization via phosphatidylinositol 3-kinase/Akt-dependent hypoxia-induced factor-1 alpha reactivation in mice of advanced age. Circulation 2010;122:707-716. 